\title{
CFD study of fluid flow changes with erosion
}

\author{
Alejandro López ${ }^{a, *}$, Matthew T. Stickland ${ }^{\mathrm{a}}$, William M. Dempster ${ }^{\mathrm{a}}$ \\ ${ }^{a}$ Department of Mechanical and Aerospace Engineering, Strathclyde University, Glasgow, \\ United Kingdom
}

\begin{abstract}
For the first time, a three dimensional mesh deformation algorithm is used to assess fluid flow changes with erosion. The validation case chosen is the Jet Impingement Test, which was thoroughly analysed in previous works by Hattori et al [1, Gnanavelu et al. in [2, 3, Lopez et al in [4] and Mackenzie et al in [5. Nguyen et al showed the formation of a new stagnation area when the wear scar is deep enough by performing a three-dimensional scan of the wear scar after 30 minutes of jet impingement test in [6] . However, in the work developed here, this stagnation area was obtained solely by computational means. The procedure consisted of applying an erosion model in order to obtain a deformed geometry, which, due to the changes in the flow pattern lead to the formation of a new stagnation area. The results as well as the wear scar were compared to the results by Nguyen et al [6] showing the same trend. OpenFOAM $₫$ was the software chosen for the implementation of the deforming mesh algorithm as well as remeshing of the computational domain after deformation. Different techniques for mesh deformation and approaches to erosion modeling are discussed and a new methodology for erosion calculation including mesh deformation is developed. This new approach is independent of the erosion modeling approach, being applicable to both Eulerian and Lagrangian based equations for erosion calculation. Its different applications such as performance decay in machinery subjected to erosion as well as modeling of natural erosion processes
\end{abstract}

\footnotetext{
${ }^{*}$ Corresponding author

Email address: alejandro.glasgow@gmail.com (Alejandro López )
}

Preprint submitted to Computer Physics Communications

January 20, 2018 
are discussed here.

Keywords: Erosion, OpenFOAM, multiphase, discrete phase model, Fluid Surface Interaction, Mesh Deformation

\section{Introduction}

Erosion is responsible, amongst others, for destroying a wide variety of equipment and causing vast losses in all kinds of industries. For over 50 years, engineers have been trying to understand the process and, as a result, a large number of scientific papers have been published on this subject. Most of these authors have captured their very own and specific ideas about the way erosion mechanisms work as well as the equations to predict wear in a number of different geometries. Meng and Ludema [7] carried out a broad literature review of more than 5000 papers dating from 1957 to 1992. In their article they identified 28 separate erosion models out of the almost 2000 existing empirical models, a fact which exemplifies the poor agreement between authors on this subject. One of the few theories on which there seems to be some kind of agreement describes two mechanisms acting together to produce the wear scar: cutting and deformation wear. When particles hit the surface and they tear material away with them in a cutting action, it is called cutting wear. This mechanism is the predominant one for ductile materials and particles impinging at low angles of attack with respect to the surface being eroded. Alternatively, several particles might impact on the same place transferring some of their kinetic energy to the surface in the form of hardening work [8. According to this theory, in a given collision with the target material, as soon as the particle contacts the surface, stress concentrations appear as a result of the elastic deformation that takes place. If these stresses are not over the elastic limit of the target material, and also leaving aside fatigue damage effects, they should cause no deformation. However, if the elastic limit is reached, plastic deformation will occur at the location of the maximum stress. The repeated impacts then create a plastically deformed layer that will deform further upon repetition of the particle 
collisions. This deformation causes hardening and increases the elastic limit in that region turning the material harder and more brittle until it reaches a point where it can no longer be plastically deformed. Eventually, upon further load, pieces of the material's surface separate from the target and are carried away by the fluid. This hypothesis was studied by Davies in [9], and Van Riemsdijk and Bitter in [10] and then adopted by several authors [11, 8, 12, 13, 14. This mechanism is called deformation wear and it predominates for high angles of impingement and in brittle materials. Erosion by solid particle impingement, however, is just one of the types of erosion investigated and to which the deformation algorithm can be coupled. Another recurrent erosion type, especially in natural processes, is described in [15], [16], 17] and [18. This type of erosion is a consequence of erodible bodies moving in viscous fluids which results in a purely fluid-mechanical erosion driven by the fluid's shear stress acting on the eroding boundaries. In processes such as erosion-corrosion, pure fluid erosion could play a role in increasing material losses once the outer layers of the target are corroded, thus increasing the total mass losses and possibly accounting for synergistic effects. Synergy has been defined as the additional wear rate experienced by the target material when both erosion and corrosion occur at the same time. The synergistic effect can be calculated by substracting the pure erosion and corrosion rates from the combined erosion-corrosion wear rate [19]. This can be represented in the form of equation 1 . Where $\mathrm{T}$ is the total wear rate, $\mathrm{E}$ and $\mathrm{C}$ are the pure erosion and corrosion wear rates respectively and $\mathrm{S}$ is the synergistic effect.

$$
S=T-(E+C)
$$

By means of a combination of both types of erosion (purely fluid and solid particle erosion), a new approach for calculating erosion-corrosion scars could be derived, including the corrosion rate of the material in addition to fluid erosion as well as solid particle erosion. In order to acquire a better understanding of the erosion process a mesh deformation algorithm is of the utmost importance. 
This algorithm enables knowing how erosion changes the shape of the domain, how the fluid flow changes with it and its effect on the erosive process. In addition, coupling the algorithm with different erosion approaches could provide new insights into other erosive processes.

\section{Erosion calculation in an Eulerian-Lagrangian frame}

In this approach, previously discussed in 4, the Eulerian equations are solved for the fluid phase and Newton's equations (2, 3) are integrated for the particles constituting the Lagrangian phase.

$$
\begin{gathered}
m_{p} \frac{d u_{p}}{d t}=F_{p} \\
\frac{d x_{p}}{d t}=u_{p}
\end{gathered}
$$

Where $m_{p}$ and $u_{p}$ are the mass and velocity of the particle, $F_{p}$ represents the forces acting on the particle and $x_{p}$ the position of the particle.

Impingement information, such as impact velocity and impact angle, is gathered by a function as particles hit the walls of the geometry. This information is introduced in an erosion model implemented in OpenFOAM in order to obtain an erosion field. The erosion formula chosen for comparison is the one developed by Tabakoff et al in [20].

\section{Eulerian phase steady-state}

An equivalent domain to the one used by Nguyen et al in [6] was set up. The mesh was composed of 2057316 elements, mainly hexahedral and the boundary types used are shown in figure 1. Convergence criteria were satisfied for the fluid phase when the residuals fell below $10^{-4}$ with second order schemes. The results of the steady state simulation for velocity and pressure are shown in figures 2 and 3 respectively 


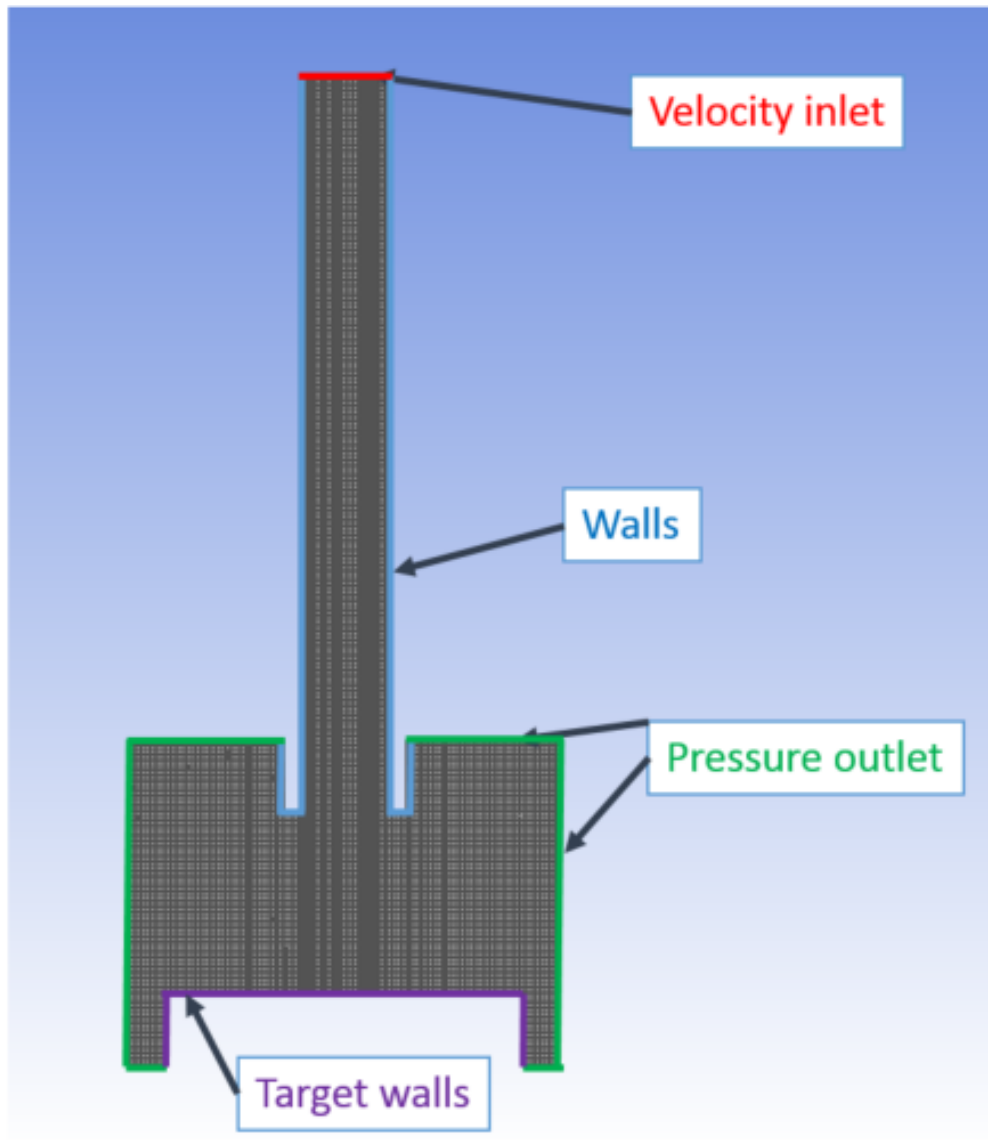

Figure 1: Mesh slice and boundary types equivalent to the ones used by Nguyen et al in 6 


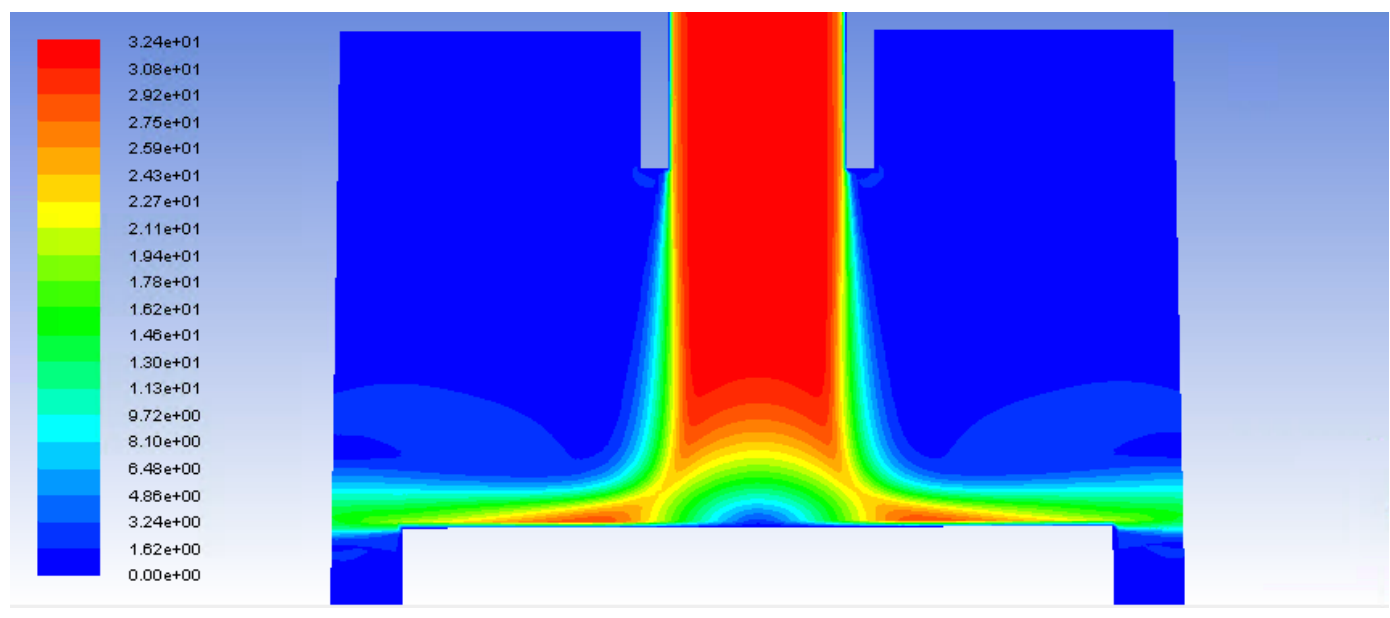

Figure 2: Velocity contours of the uneroded geometry $\left(\frac{m}{s}\right)$

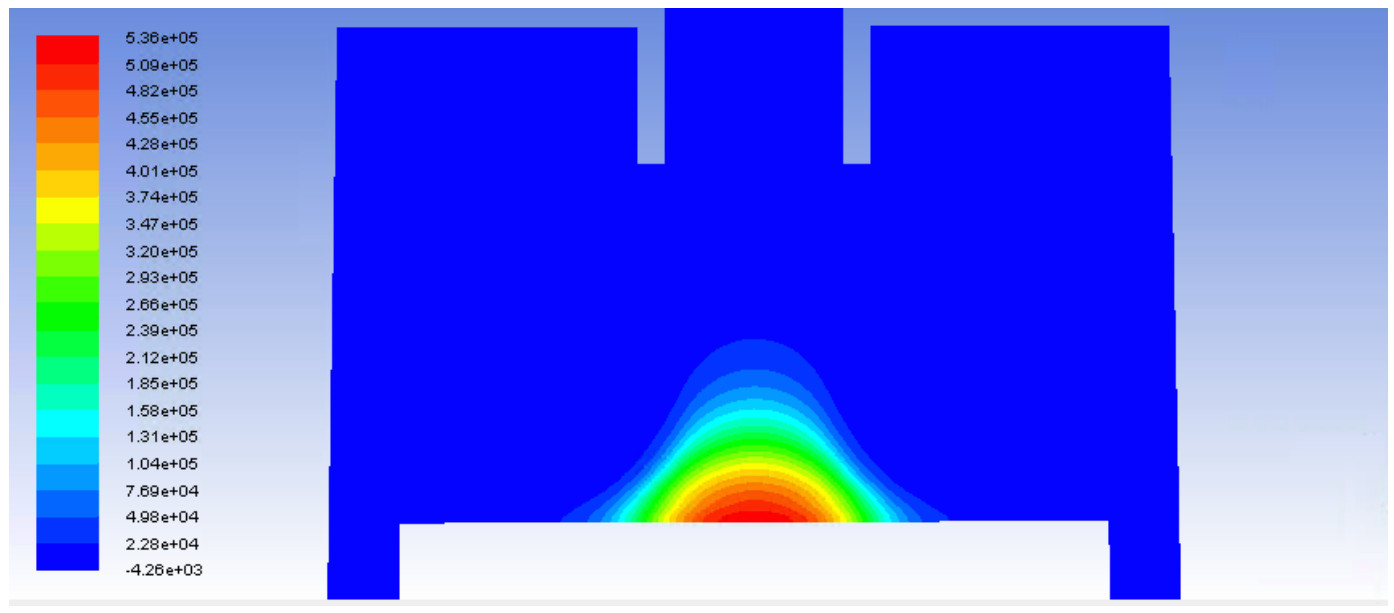

Figure 3: Static pressure contours of the uneroded geometry $(P a)$ 
Different turbulence models were assessed for the jet impingement. The first one was the $k-\epsilon$ model [21, which has repeatedly been used to predict erosion in the jet impingement test as well as other configurations [6, 22, 2, $[3$, and the second one was the $k-\omega S S T$ model [23]. The $k-\epsilon$ model is a semiempirical model composed of two equations offering reasonable accuracy at an acceptable computational cost. These reasons make it very popular in industrial applications and over a wide range of flow regimes 24]. On the other hand, the Shear Stress Transport $(S S T) k-\omega$ brings together $k$ - $\omega$ 's robust and accurate formulation near the walls with $k-\epsilon$ 's free-stream independence in the far field [24. In a recent publication, the validity and accuracy of some of the most widely used turbulence models (including $k-\epsilon$ and $k-\omega S S T$ ) was assessed by Mackenzie et al in [5] against Particle Image Velocimetry of the fluid phase, concluding from the profiles of the velocities close to the impingement area that the most widely used one, which is the $k-\epsilon$ model, is able to capture the general trend of the axial and radial velocities at a reasonable computational cost. With the known limitations of two-equation turbulence models, the $k-\epsilon$ model seems like a reasonable approach, given that it is the general trend of the velocity vectors, what will define the form that the wear scar takes. Additionally, the $k-\epsilon$ model was also used by Nguyen et al after validating its accuracy in [6] and, given that the aim in this work is to reproduce the results obtained experimentally in that previous study, the same turbulence model is used here.

\section{Discrete phase modeling}

Once the steady-state values for the main flow field variables were obtained, these were set as the initial conditions for the transient simulations. Particle tracking is carried out by numerically integrating the sum of the forces acting on the particles in order to obtain velocities and positions. The force balance according to equation 2 for the case considered is shown in equation 4

$$
F_{p}=m_{p} \frac{d u_{p}}{d t}=F_{D}
$$


No forces other than the drag force were considered since their values relative to the drag force were negligible. Several additional simulations were run incorporating other forces such as added mass, gravity etc with no significant differences in the results for the averages of the velocity and impact angle on the target. The drag force $\left(F_{D}\right)$ on spherical particles takes the form of equation 5 and the drag coefficient is obtained from equation 6 , which is composed of three parts corresponding from top to bottom of the equation to the Stokes' Law region, the Transition region and the Newton's Law region respectively.

$$
\begin{gathered}
F_{D}=m_{p} \frac{18 \mu}{\rho_{p} d_{p}^{2}} \frac{C_{D} R e_{p}}{24}\left(u-u_{p}\right) \\
C_{D}=\left\{\begin{array}{lr}
\frac{24}{R e_{p}} & : R e_{p}<1 \\
\frac{24}{R e_{p}}\left(1+0.15 R e_{p}^{0.687}\right) & : 1 \leq R e_{p} \leq 1000 \\
0.44 & : R e_{p}>1000
\end{array}\right.
\end{gathered}
$$

Some of the features corresponding to the transient simulation are shown in Table 1:

\begin{tabular}{|l|c|r|}
\hline Variable & Units & Value \\
\hline \hline Time-step & $s$ & $1 * 10^{-05}$ \\
\hline Number of time-steps & - & $1 * 10^{6}$ \\
\hline Particle diameter distribution & - & Rosin-Rammler $[25$ \\
\hline Particle diameter maximum Value & $m$ & $180 * 10^{-6}$ \\
\hline Particle diameter minimum Value & $m$ & $125 * 10^{-6}$ \\
\hline Coupling between phases & - & One-way \\
\hline Forces & - & Drag \\
\hline Drag coefficient & - & nonSphereDrag \\
\hline Phi (Sphericity coefficient) & - & 0.58 \\
\hline Particle density & $\frac{K g}{m^{3}}$ & 2400 \\
\hline
\end{tabular}

Table 1: Transient simulation features

Since the particles used in the experimental work by Nguyen et al [6] were 
not spherical, a sphericity coefficient $p h i$ was set for the non-spherical drag equation.

\section{Erosion calculation and mesh deformation in OpenFOAM}

In the following section, the procedure followed to deform the mesh according to the erosion field is explained in detail. First, the application used to compute the erosion field is described. Thereafter, the procedure consists of a series of operations with the erosion field until the desired output is reached. It is after this transformations that the mesh boundary will be deformed by means of an extension of an application called deformedGeom, which allows moving the mesh boundary according to a field of vectors stored at each time step.

\subsection{Erosion modelling in OpenFOAM}

In order to calculate erosion in OpenFOAM, one of the available Lagrangian libraries was extended. One of the classes OpenFOAM provides the users with is the CloudFunctionObjects. This is a templated library that adds additional capabilities to the Lagrangian. The main template discussed here is called particleErosion. This set of files create the particle erosion field on the userspecified patches. The result is a field of scalars, which, at each face, will be the sum of the volume eroded by all the particle hits. Impingement information, such as impact speed and impact angle, is gathered as particles hit the walls of the geometry. These are then introduced in the desired formulae, when different erosion models, as in this case, are implemented inside the library. Functions within the library give access to the particle variables at the moment of impact with the target so that these values can be introduced in the erosion formula in order to compute the erosion field.

One of the common issues that was addressed relates to the number of impacts needed to obtain a good average for the erosion field. In order to calculate how many impacts on the target are needed for an accurate representation of the impact probability on the whole sample the transient simulation is used. 
Once the steady state in the transient simulation is reached, i.e., the number of particles within the control volume doesn't change or fluctuates around a number, the parameters that allow calculation of the size of the sample needed are obtained. Three new fields are also computed which give some insight into how the averages are evolving during the simulations. These can be written to memory at any time step. For the particular case of a discretised plane, as in the boundary being impinged by particles, the total area (surface of the target) is divided into a set of faces. This means that, as the simulation progresses, a mean and a standard deviation can be calculated for each of the faces of the boundary. By running a transient simulation, a time for which the mean values and the standard deviation stop changing can be found. Once that simulation has been studied and the mean and standard deviation values satisfying the criteria are met, two meaningful values for each of the faces (the mean and the standard deviation) are produced. With these two values and the formula used for calculating the size of a sample, the minimum number of particles necessary for a good average can be obtained. In order to calculate the size to obtain a good average of the erosion field first, the level of confidence has to be specified. In this case, if this level is set to be $99 \%$, looking on the table of the Normal Distribution it can be inferred that $z_{\frac{\alpha}{2}}=2.576$. In addition to that, the error needs also be specified. For this variable, a 5 per cent of the mean velocity at each cell is chosen. The procedure is illustrated equations 7 and 8 , of which the latter is used for calculating the sample size for each of the faces of the boundary.

$$
\begin{gathered}
\delta=\frac{z_{\frac{\alpha}{2}} \sigma}{\sqrt{n}} \\
n=\left(\frac{z_{\frac{\alpha}{2}} \sigma}{\delta}\right)^{2}
\end{gathered}
$$

Where $\delta$ is the maximum error of the estimate or the half-width of the confidence interval, $\mathrm{n}$ is the size of the sample and $\sigma$ is the standard deviation [26]. 


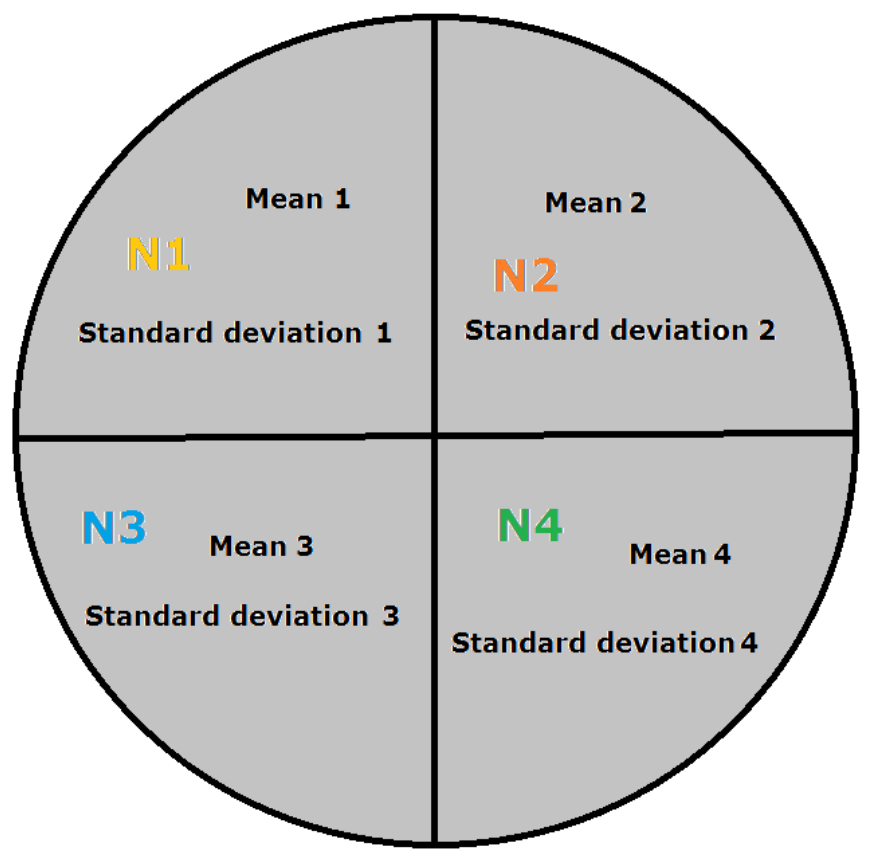

Figure 4: Representation of a circular domain divided in four faces, where N1, N2, N3 and N4 are the sample sizes to be obtained for each of the faces 
With the statistical analysis outlined in this section the minimum number of impacts needed to obtain both velocity and impact angle averages with a certain degree of confidence can be calculated. In order to do this, 10 seconds of erosion were simulated for the case studied by Nguyen et al [6]. Initially, a visual inspection of the results for different times, showed that the wear scar variations after the first second of simulation were negligible, as shown in figure 5 , where the wear scar was obtained using the formula developed by Tabakoff et al [20]. These figures show how the contours only increase their values without changing the shape of the scar from the first second of simulation. The formula used in the calculations is shown in equation 9, where $\epsilon$ is equal to the erosion per unit mass of particles, $\beta_{1}$ is the relative angle between the particle trajectory and the surface, $\beta_{0}$ the angle of maximum erosion, $V_{1}$ the velocity of the particle and $R_{T}$ the tangential restitution ratio. Values for the empirical constants $K_{1}, K_{1} 2$ and $K_{3}$ were calculated for aluminium in [20] and their values are $1.56988 e^{-} 6$, 0.3193 and $2.0 e^{-} 12$ respectively.

$$
\begin{aligned}
& \epsilon=K_{1} f\left(\beta_{1}\right) V_{1}^{2} \cos ^{2} \beta_{1}\left(1-R_{T}^{2}\right)+f\left(V_{I} N\right) \\
& R_{T}=1-0.0016 V_{1} \sin \beta_{1} \\
& f\left(\beta_{1}\right)=\left[1-C K K_{1} 2 \sin \left(\frac{90}{\beta_{0}}\right) \beta_{1}\right]^{2} \\
& C K= \begin{cases}1 & \beta_{1} \leq 3 \beta_{0} \\
0 & \beta_{1}>3 \beta_{0}\end{cases}
\end{aligned}
$$

Since one of the aims is to optimise the computational time invested in running the simulation, the results between time $0 \mathrm{~s}$ and $1 \mathrm{~s}$ were analysed every $0.1 \mathrm{~s}$ of simulation. In addition to this, with the aid of the statistical fields developed, the minimum number of impacts required per face were calculated. The results after 10 seconds of simulations were used in order to calculate this minimum number of impacts with a confidence level of $99 \%$. The number of particles released after 10 seconds was 1 million. Additionally, an application was compiled in order to sum up the minimum number of impacts required at each face for the impact angle and impact velocity averages. Of the two numbers obtained, the highest one was chosen as the minimum number of impacts. The 

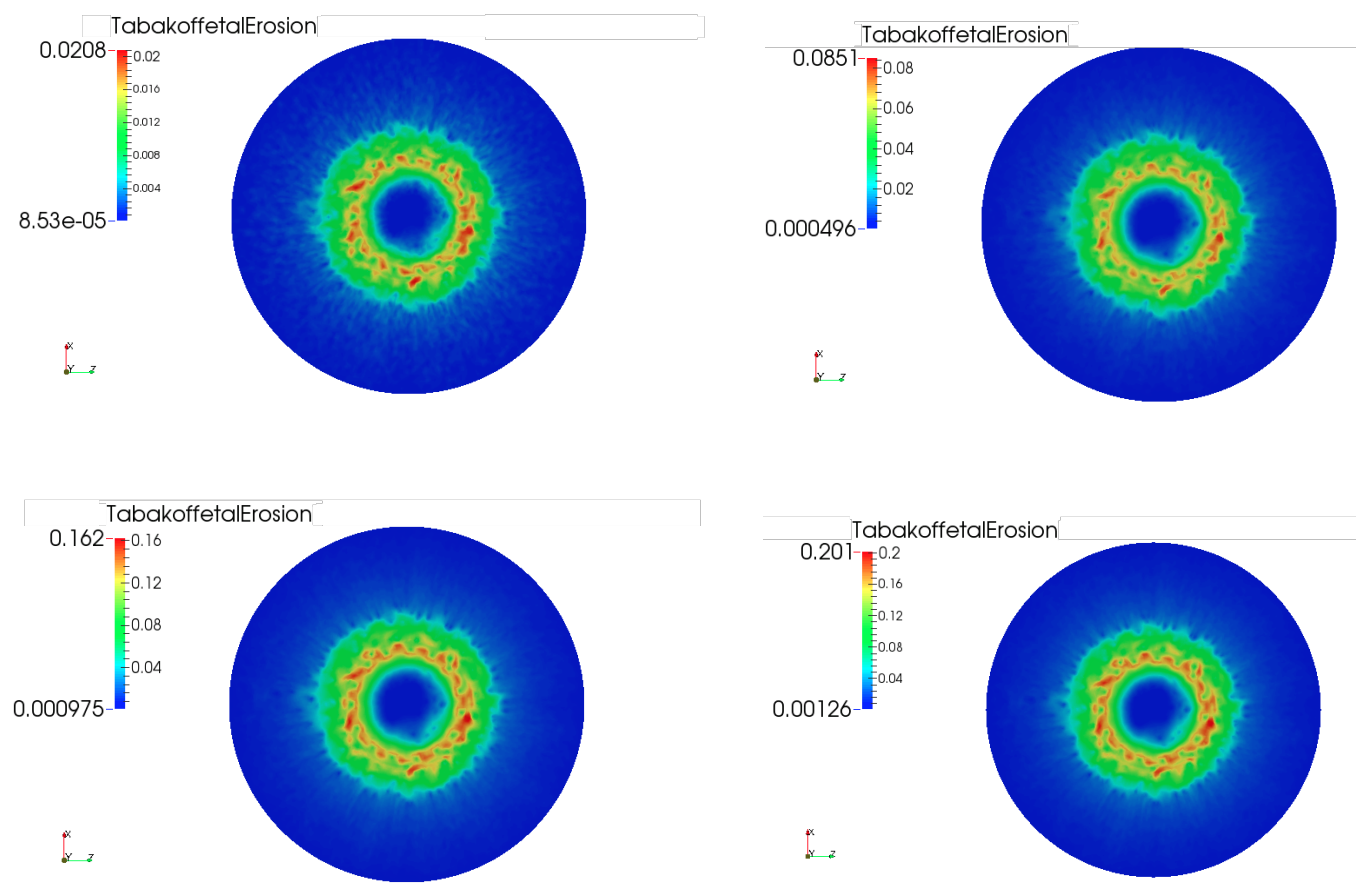

Figure 5: Contours of erosion per unit mass of impacting particles at 4 different simulation times. From left to right and from top to bottom: 1, 4, 8 and 10 seconds 

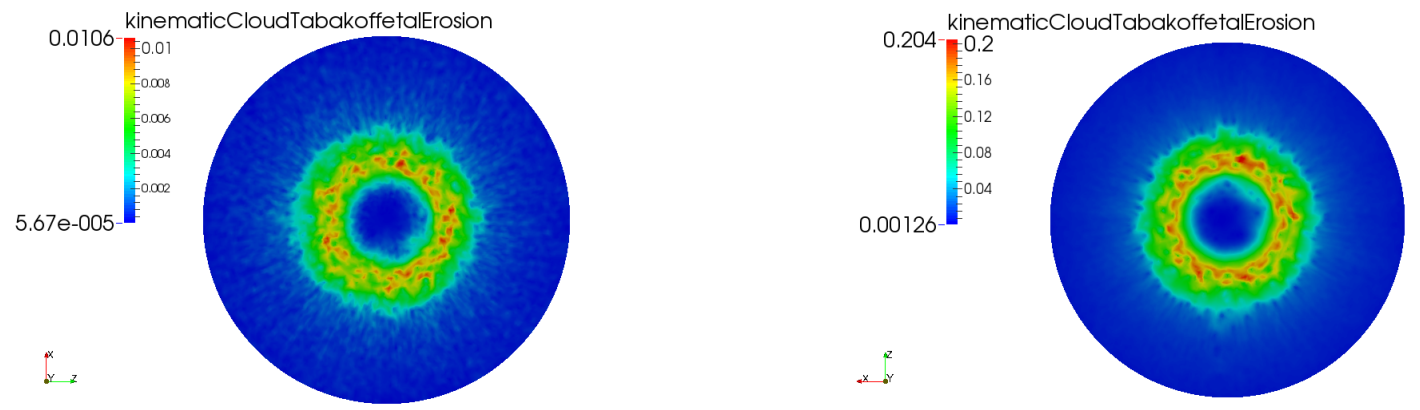

Figure 6: Contours of erosion per unit mass of impacting particles at 0.5 and 10 seconds of simulation

value obtained for the simulation was 48677 impacts (or $0.48677 \mathrm{~s}$ ). However, this value only accounts for the time at which the particles are released. Thus, the average time that a particle spends inside the domain was added to the 0.48677 seconds. The calculation of the average time a particle spends in the domain was obtained from the log of OpenFOAM's particle variables and estimated to be 0.02 seconds for this simulation. It was after this simulation time that the number of particles within the domain fluctuated around a constant value. Therefore, the total time was rounded up to 0.5 seconds of simulation. A qualitative comparison between the erosion contours at time 0.5 seconds and 10 seconds is shown in figure 6. Figure 7 shows a comparison of the normalised erosion ratio between the same two time-steps plotted along the radius of the target. The average difference between lines is $1.19 \%$, which validates the statistical accuracy of the erosion contours at $0.5 \mathrm{~s}$.

However, this process can be made in two different ways: an escape condition can be set up at the target's boundary (as done by Gnanavelu et al in [2, 3]) in which the particles are eliminated from the domain as soon as they reach any of the faces of the boundaries, or a rebound model may be chosen (or implemented) in which rebound coefficients are defined for the particles. If the latter is used, the impact velocity average is radically different, as can be observed in images 


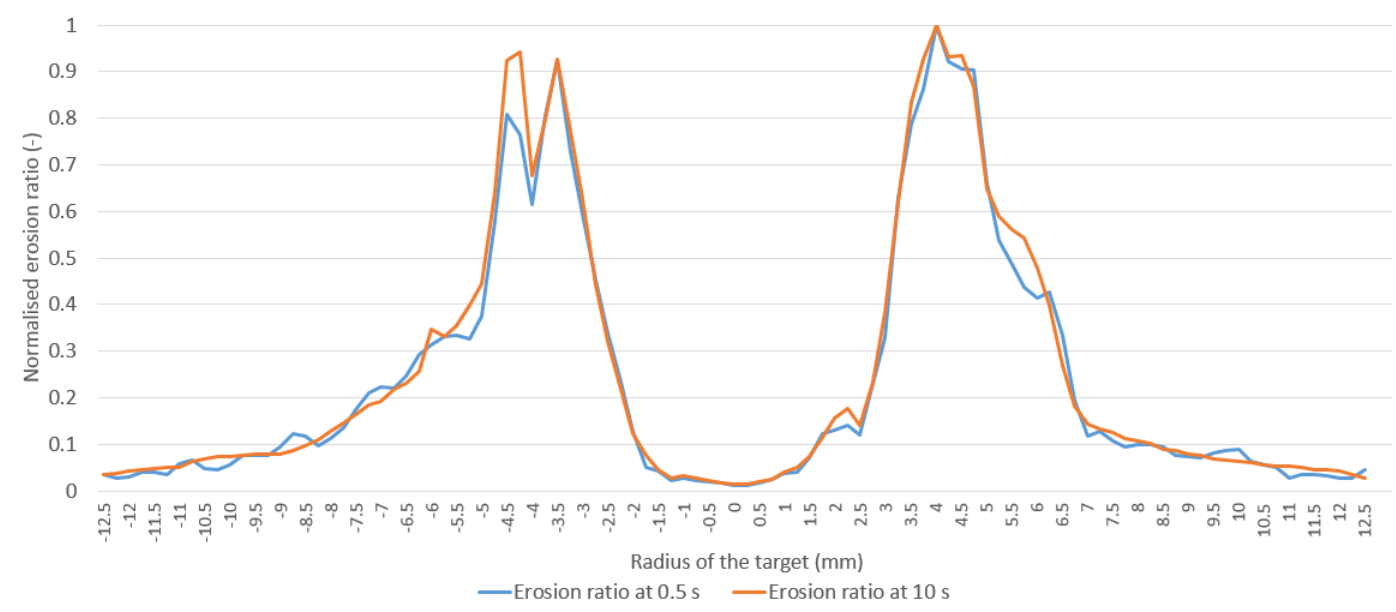

Figure 7: Comparison of the normalised erosion ratio over the radius of the probe $(\mathrm{mm})$

8 and 9 since the first impacts have the highest kinetic energy and a big part of it is lost thereafter.

Differences are also spotted in the mean angle of impingement and also, the number of impacts is around $1.5 * 10^{5}$ times higher, as can be observed in images 10, 11, 12 and 13 .

\subsection{Influence of the rebound model}

The computational erosion obtained by Nguyen et al in [6] incorporated a different erosion model than the default one in OpenFOAM. The model used was developed by Forder et al in [27. Forder's model was implemented in OpenFOAM and a $10 \mathrm{~s}$ simulation was obtained for each model. The erosion contours obtained after 10 seconds are shown in figure 14 . As can be observed, the wear scars obtained with both models are practically identical. The only difference between both scars relates to the magnitude of the erosion. This indicates that a simple model, which would also be less computationally expensive, would be sufficient to calculate erosion with enough accuracy for this configuration.

For validation of the mesh deformation algorithm a total of 10 seconds of transient simulation is set up and the fields are monitored every 0.2 seconds. Values of the fields at 10 seconds are used for calculation of the number of 


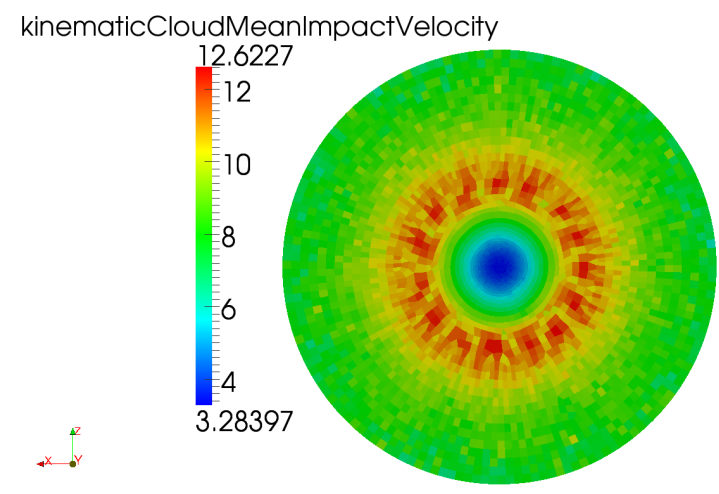

Figure 8: Face-wise impact velocity average $\left(\frac{m}{s}\right)$ after 10 seconds with an escape condition at the target's boundary

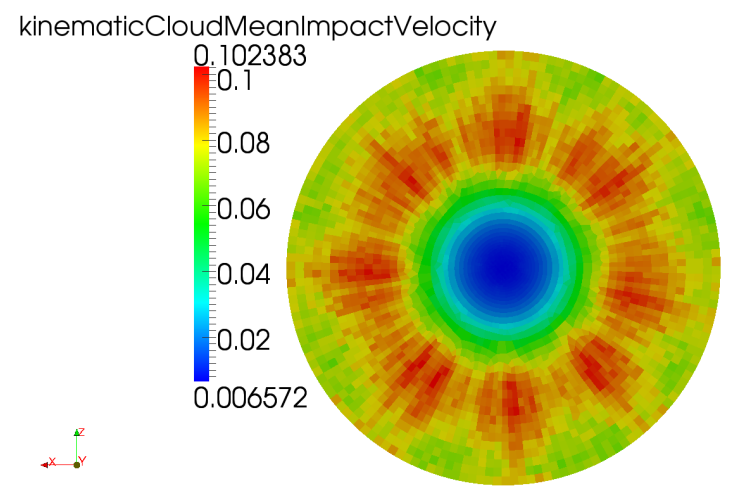

Figure 9: Face-wise impact velocity average $\left(\frac{m}{s}\right)$ after 10 seconds with Forder's 27] rebound model at the target's boundary 


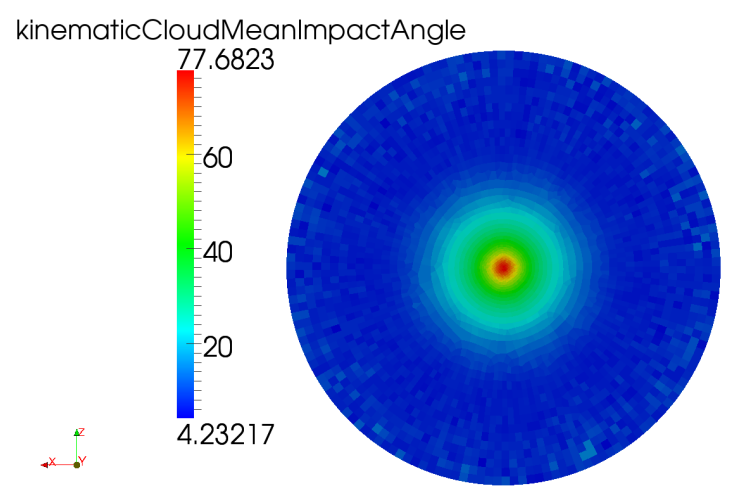

Figure 10: Face-wise impact angle average (degrees) after 10 seconds with an escape condition at the target's boundary

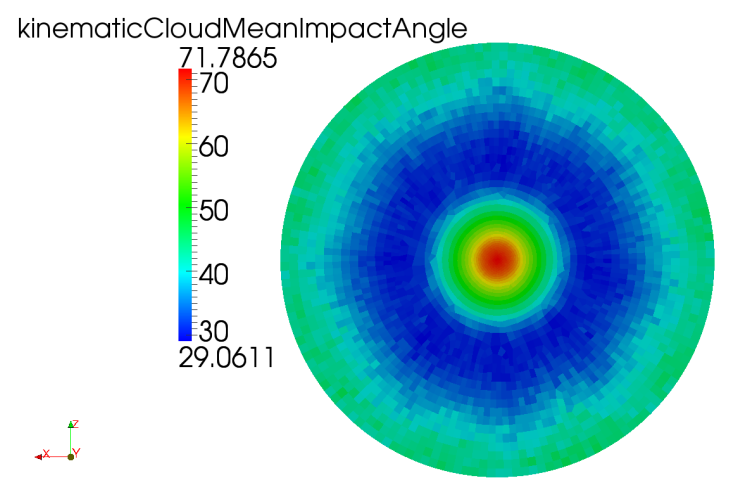

Figure 11: Face-wise impact angle average $\left(\frac{m}{s}\right)$ after 10 seconds with Forder's 27 rebound model at the target's boundary 


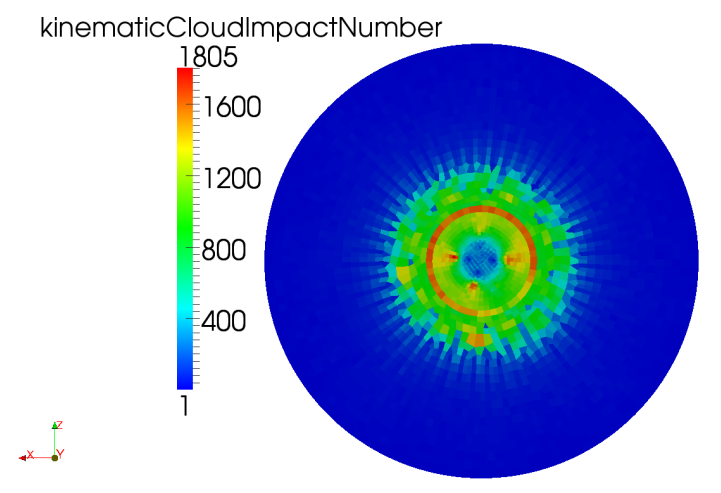

Figure 12: Face-wise impact number after 10 seconds with an escape condition at the target's boundary

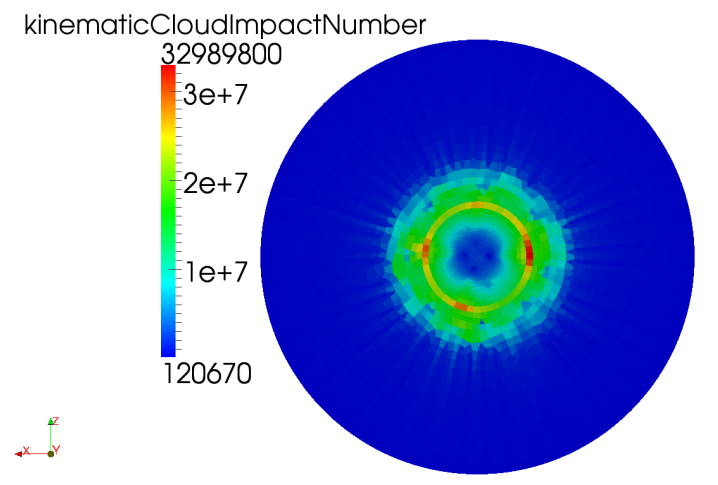

Figure 13: Face-wise impact number after 10 seconds with Forder's 27] rebound model at the target's boundary 

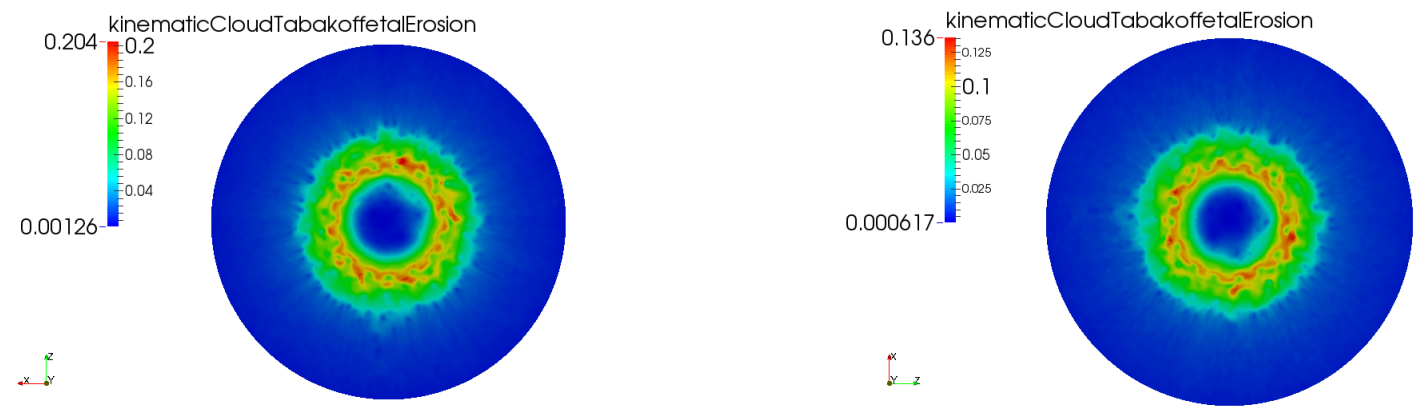

Figure 14: Contours of erosion per unit mass of impacting particles for the same erosion model [20] and different rebound models. Forder et al 27] (left) and OpenFOAM's default rebound model (right)

impacts and the velocity and angle averages will be taken from the case with the rebound model developed by Forder et al [27] that was also implemented in OpenFOAM. This rebound model was also the same one used by Nguyen et al in [6].

\subsection{Mesh deformation according to erosion}

Once the erosion field has been calculated, the mesh deformation can be accomplished through modification of the surface and by moving the boundary points. Once steady state erosion is reached, the wear scar varies in magnitude but its location and shape remain unchanged. Hence, the erosion field can be subjected to an amplification which would be the equivalent of advancing the simulation in time. This seems reasonable provided that, after a certain number of impacts which can be calculated through sample size determination, the shape of the scar does not vary significantly until the flow does. This so called steady state erosion has been observed before by Huttunen-Saarivirta et al and Head et al among others in [28, 29]. The erosion field calculated contains a value at each of the boundary faces and it has a value of zero for all the internal cells. A field that contains all the surface area vectors is then created. A surface area vector is defined as a vector that points outside of the cell, it is orthogonal to 
the face and its magnitude is the area of the face. At the boundaries, the face area vectors will point outside of the domain and these will give the direction for the point displacement. As the magnitude of the displacement of the mesh points will be equal to the magnitude of the erosion, unit normal vectors are required. By dividing the face area vectors by their magnitude, the unit face area normal vectors are obtained as defined in equation 10 .

$$
\hat{\boldsymbol{u}}=\frac{\boldsymbol{u}}{\|\boldsymbol{u}\|}
$$

A field of vectors orthogonal to each of the boundary faces is stored to memory. This field will be used to multiply it by the erosion field of scalars. The final step before being able to move the mesh points is to interpolate the field values contained at each face of the boundaries to each point of those faces using an inverse distance weighting interpolation algorithm. The same methodology for mesh deformation was applied in combination with dynamic meshing. In doing this, a new solver for erosion calculation was created in which the mesh is updated according to the user's instructions and after mesh deformation, the flow field is recalculated, thus, avoiding extra computational time.

\subsection{Validation of the 3-dimensional wear scar}

An equivalent case to that one of Nguyen et al [6] was set up for validation. Steady state results were computed first and after that, an Euler-Lagrange simulation was run in OpenFOAM in order to calculate erosion induced by solid particle impingement. The parameters of the simulation were set to be the same as the ones used by Nguyen et al in [․ The formula used for prediction of the erosion contours was developed by Tabakoff et al in 20] and implemented in OpenFOAM. This model was run alongside other models such as the ones developed by Menguturk et in [30] (also discussed in 31] and 32]) and Nandakumar et al [33] producing for all of them very similar erosion profiles and only differing in magnitude. The mesh deformation is applied after 10 seconds of simulation. 


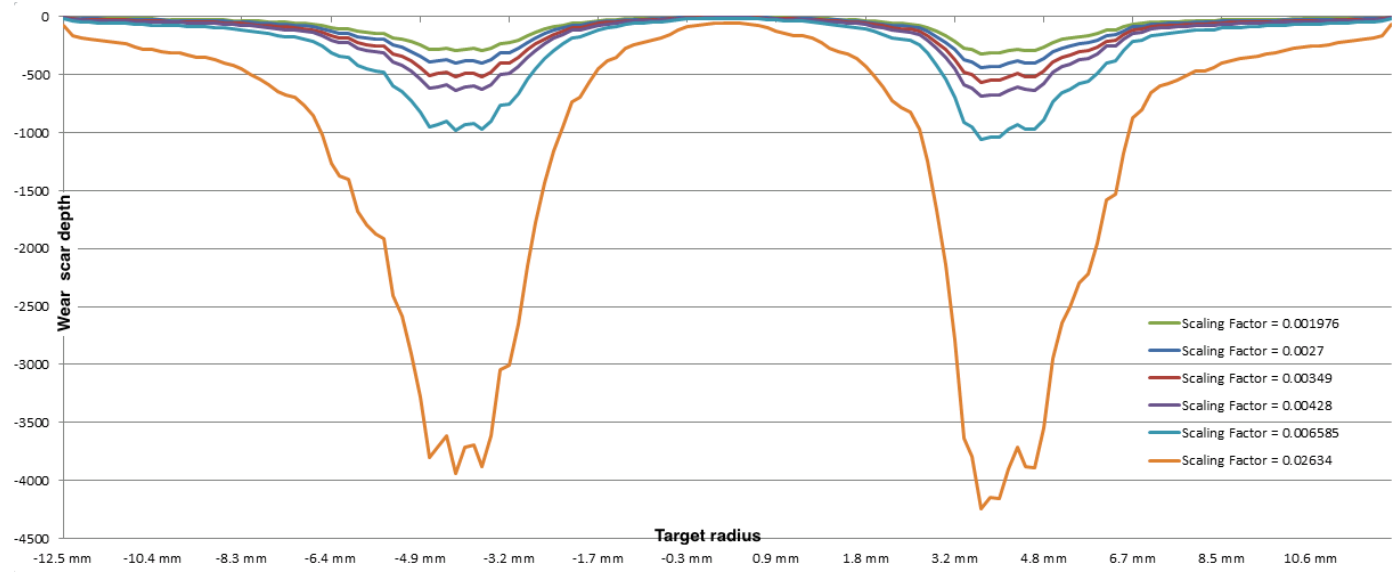

Figure 15: Wear scar profile depth comparison $(\mu m)$ along the radius $(\mathrm{mm})$ for different scaling factors

Once the erosion ratios were calculated, the scar was scaled so that the maximum depth was $542 \mu \mathrm{m}$ and the steady state was calculated again. The scaling factor which corresponds to $542 \mu m$ of depth was 0.0349 for the erosion contours obtained with the proposed formula. Additionally, different scaling factors were applied in order to analyse the evolution of the fluid flow during the steady state erosion. A radial average of the wear scars was obtained for each scaling factor and the different profiles are shown in figure 15

A quantitative comparison between the wear scar profile obtained with simulations and the ones measured by Nguyen et al is shown in Figure 16. It was also found that, as the scar progresses, the new stagnation point is captured, validating the deformation algorithm. As opposed to the work developed by Nguyen et al in [6], where the wear scar is 3D scanned and introduced into the CFD software again, the results here were obtained entirely computationally after $10 \mathrm{~s}$ of simulation. The initial contours of velocity and pressure are shown in figures 2 and 3 respectively while the same are shown in figures 17 and 18 respectively for the deformed geometry after being eroded for a value of the scaling factor of 0.00349 .

Additionally, the same two images are shown for the first four scaling factors 


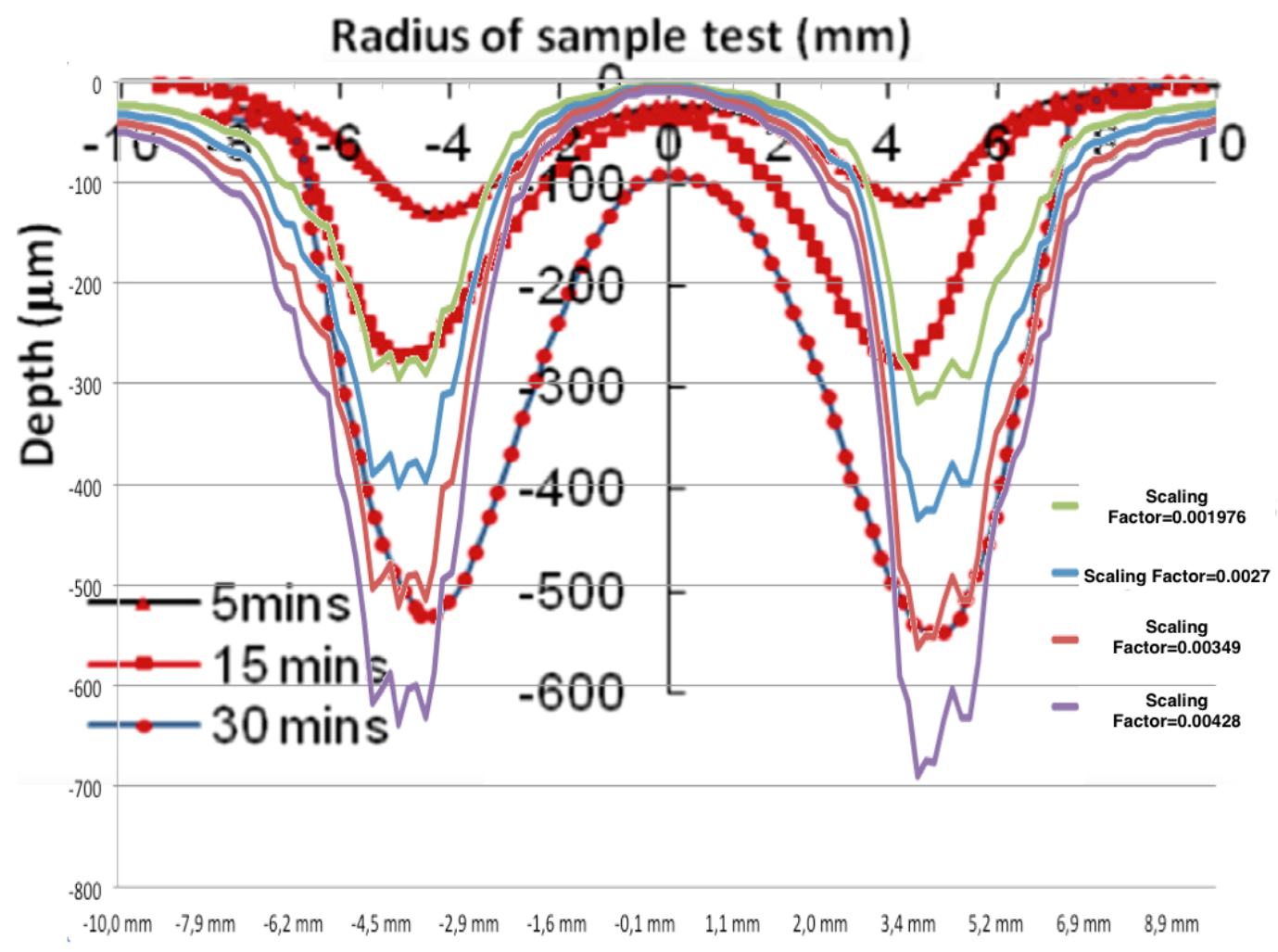

Figure 16: Wear scar profile comparison with the experimental scars measured by Nguyen et al in [6] 


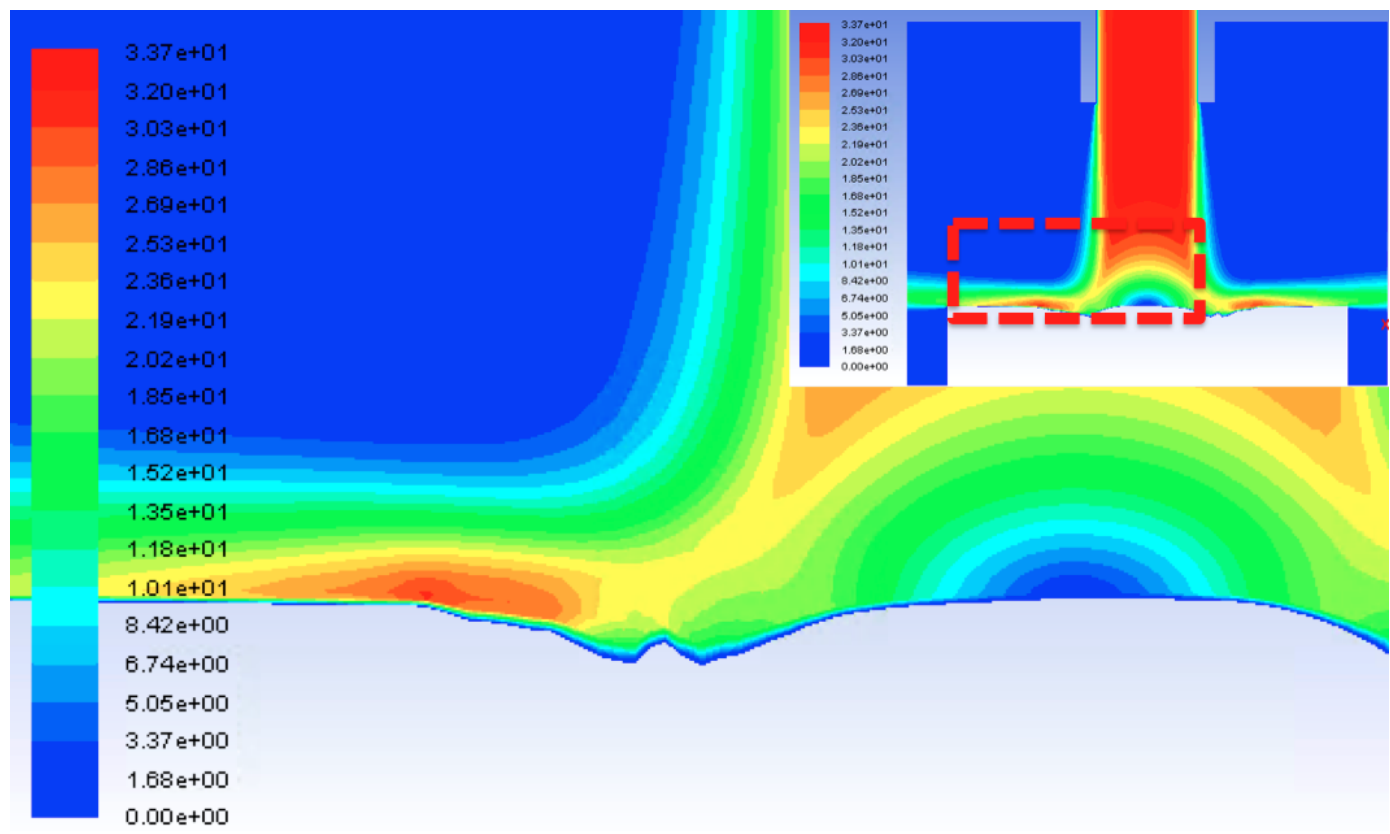

Figure 17: Velocity contours of the eroded geometry $\left(\frac{m}{s}\right)$ for a scaling factor of 0.00349

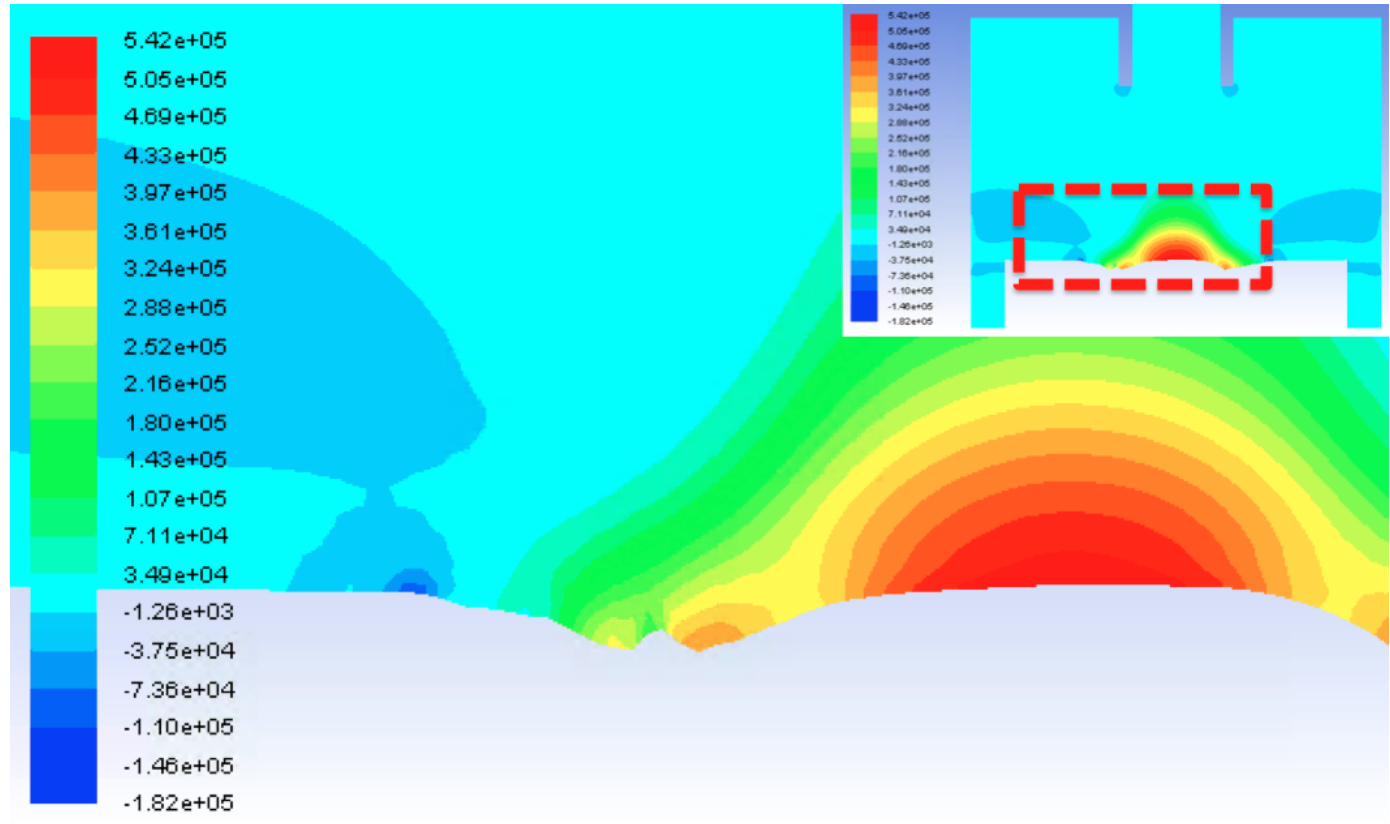

Figure 18: Static pressure contours of the eroded geometry $(\mathrm{Pa})$ for a scaling factor of 0.00349 
analysed in figure 16 .

Figure 20 shows the different surfaces obtained after the mesh deformation for the same scaling factors.

Figure 21 shows the pressure contours at the deformed surfaces for all the scaling factors with different scales for the pressure. This figure seems to indicate that the stagnation point appears even before an equivalent depth to the experiment of Nguyen et al [6]. In the computational calculation, the first appearance of the stagnation point was detected for a scar depth 1.766 times smaller than that the one reported by Nguyen et al in [6].

It is worth noting that the values of the pressure around the stagnation area increase as the wear scar progresses while the location of the stagnation point doesn't change its relative location. However, the maximum velocity generated by the new scars significantly changes its value at around the same depth analysed by Nguyen et al in [6], which corresponds to a scaling factor of 0.00349 . After that point, it fluctuates between different values, not increasing any further. Therefore, it is predicted that the fluid flow changes would be significant enough to affect particle trajectories once the wear scar is around $540 \mu \mathrm{m}$ in depth which could be confirmed by calculating erosion on the updated surfaces to see if the shape of the erosion scar changes between the different depths. These results confirm the validity of the approach for the wear scar obtained by Nguyen et al in [6] after 30 minutes of erosion.

\subsection{Time-scaling}

The proposed methodology introduces three different time-scales. These scales are optimised in order to calculate the deformed state of the geometry once eroded in the minimum amount of time possible.

\subsubsection{Lagrangian particles}

The Lagrangian time-scale is related to the time-step set in the solver in order to calculate particle trajectories accurately. In this type of simulations the time-step should satisfy a low enough Courant-number so that the trajectories 

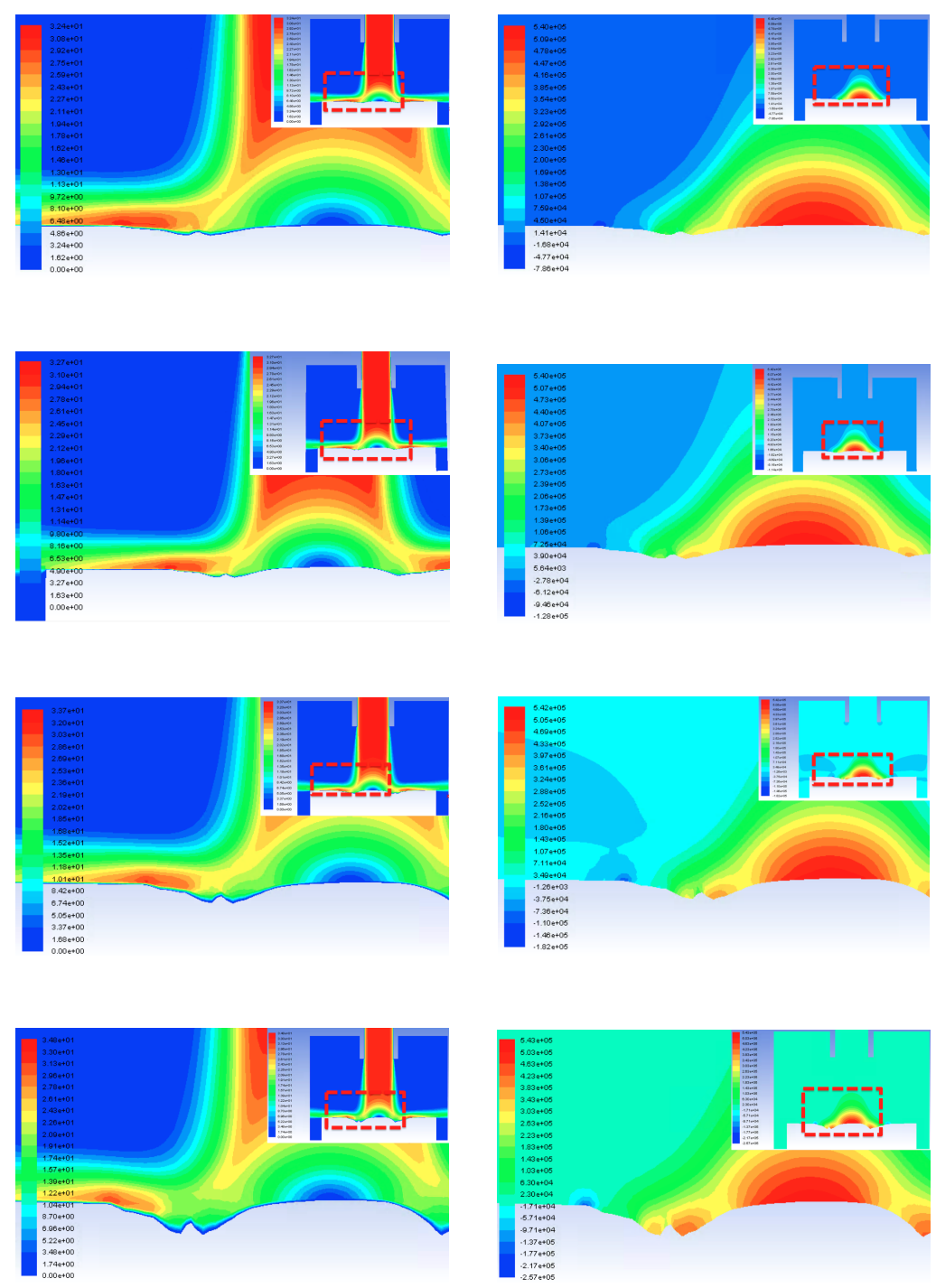

Figure 19: Velocity $\left(\frac{m}{s}\right.$, left) and pressure contours ( $P a$, right) for all the scaling factors. From top to bottom: 0.001976, 0.0027, 0.00349, 0.00428 

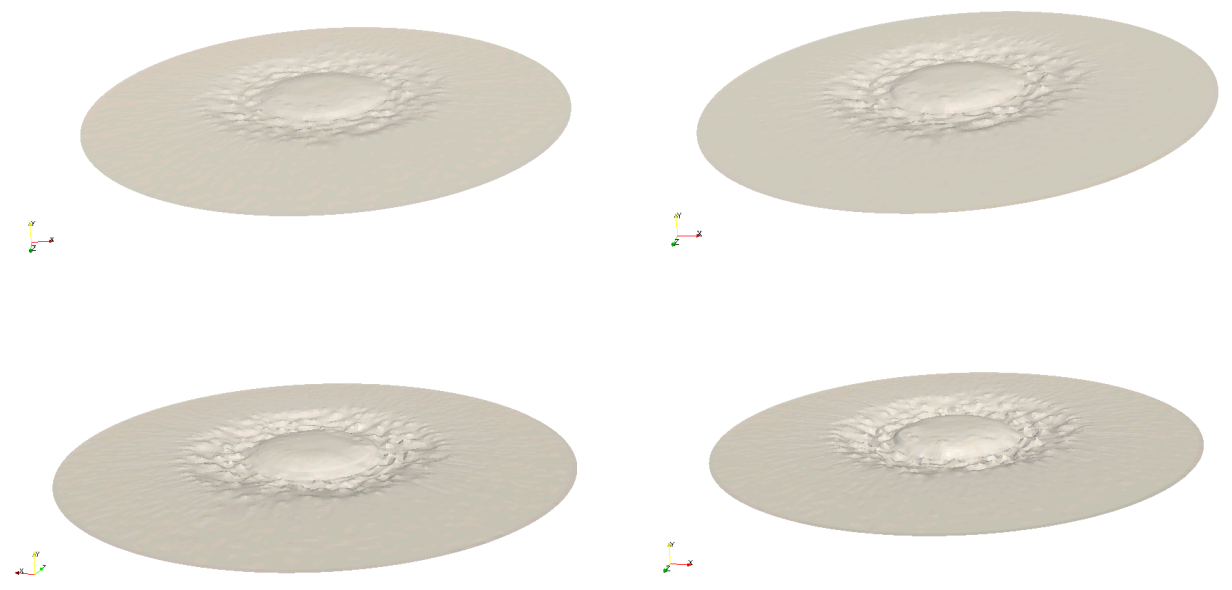

Figure 20: Surfaces obtained for all the scaling factors. From top to bottom and left to right: $0.001976,0.0027,0.00349,0.00428$

are calculated accurately. The lower the Courant-number, the more accurately these will be calculated. In theory, the Courant number should be kept below a value of 1 . In the simulations shown in this work, the Courant number was kept between 0.2 and 0.9. The Lagrangian time-step used in the simulations for validation was $1 e^{-5} s$.

\subsubsection{Erosion and mesh deformation}

The time-scale related to both erosion rate and mesh deformation, will be dependent upon the number of impacts necessary in order to calculate the wear scar with the chosen level of confidence. The mesh deformation time-scale will have the same value, as the algorithm will be applied when the wear scar is accurate enough. In this case, as it was outlined in this section, this value should be equal to 0.5 seconds.

\subsubsection{Fluid flow}

Finally, the fluid-flow time-step should have the same value as the erosion and mesh deformation one. The reason behind this is that only after the mesh is 

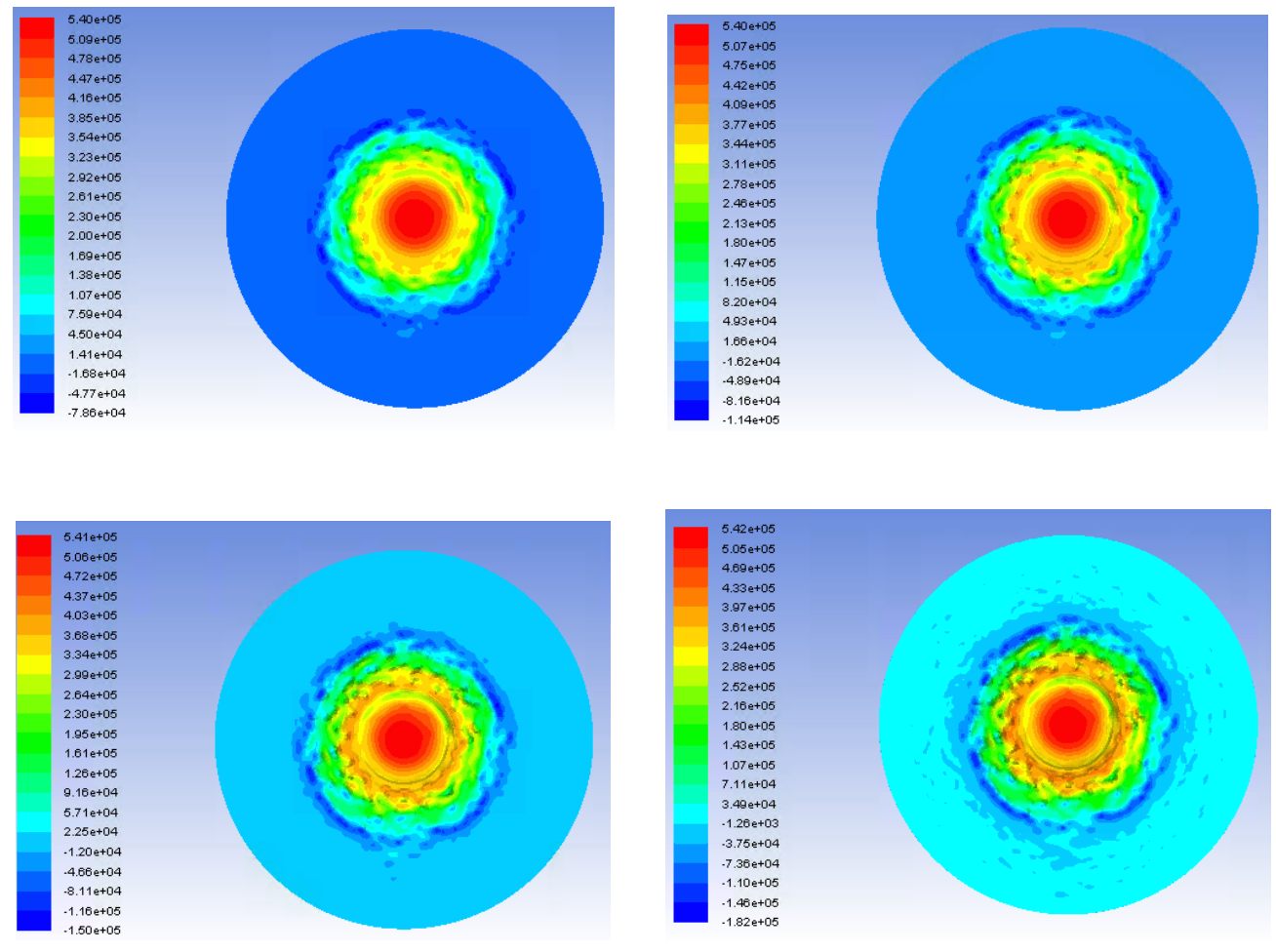

Figure 21: Pressure contours $(\mathrm{Pa})$ at the surfaces for all the scaling factors. From top to bottom and left to right: $0.001976,0.0027,0.00349,0.00428$ 
deformed, the fluid flow steady state has to be recalculated in order to compute the modified particle trajectories. Regarding the number of iterations of fluid flow in order to achieve a new steady state, this will depend on the complexity of the geometry on which erosion is calculated.

\section{Erosion calculation with a dynamic mesh solver}

In section 5.4 the mesh was refined after deformation through creation of layers between the boundary and the cells next to it. A different technique was implemented in order to avoid having an increasing size of the cells adjacent to the boundaries, which was to embed a dynamic mesh solver within the code that moves the points of these adjacent cells and creates new ones where required. Dynamic meshing with a Laplacian solver and inverse distance interpolation [34, 35] was implemented in this case although there are other schemes available for dynamic meshing as well as interpolation. Figures 22 and 23 represent two series of images of the mesh and velocity contours obtained with this code for the case studied in [6] in a smaller mesh of 230000 cells. In figure 22, it can be observed how, as deformation progresses, the whole mesh is adapted to the new shape of the deformed geometry according to the inverse distance weighting algorithm.

When the dynamic mesh was used, an additional dictionary was included where the number of fluid flow iterations after deforming the mesh was defined as well as other parameters such as the value determining when convergence was reached. In this case, that number was set to 10 and the values for determining

when convergence was reached was set to $10^{-4}$. Figure 23 shows the increasing trend of the magnitude of the velocity as the erosion scar deepens. This is due to the increasing the bend in the fluid flow which, eventually will form a new stagnation area around the wear scar. When dynamic meshing is included, it is important to set a sufficient number of iterations of the continuous phase to ensure that convergence has been reached before iterating the discrete phase. With this solver, a study of the minimum deformation necessary for a significant 

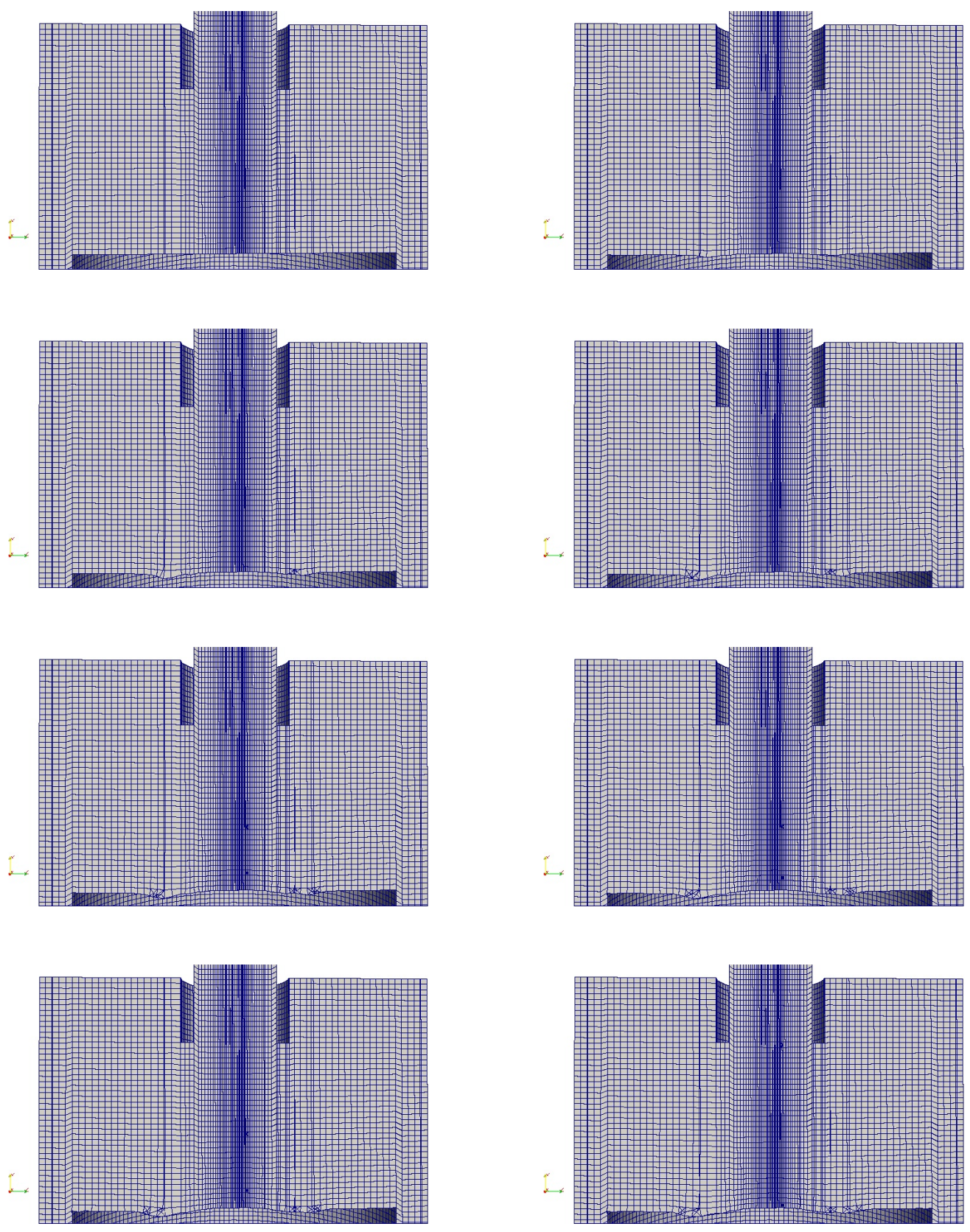

Figure 22: Progressive mesh deformation and result of the dynamic meshing 

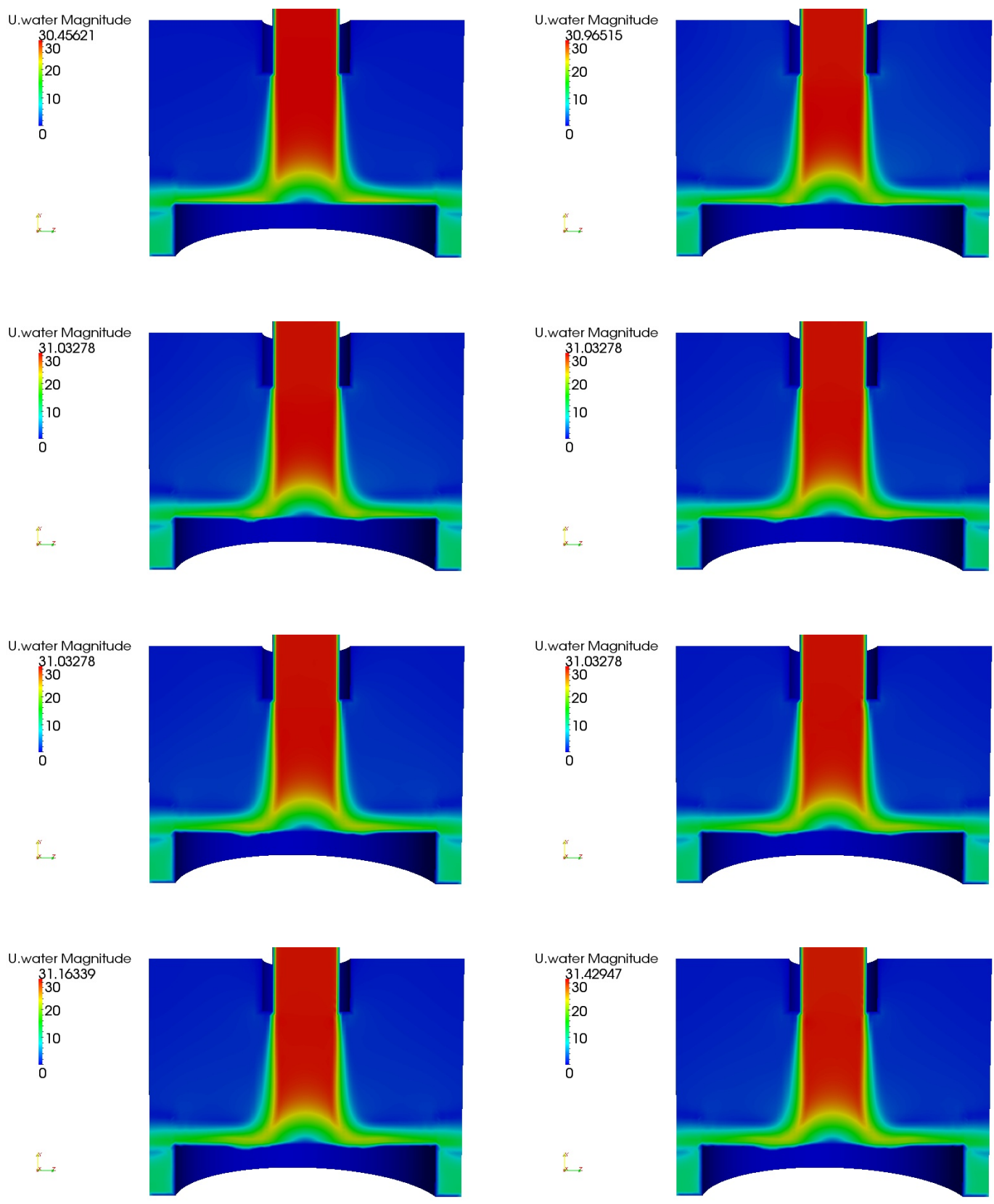

Figure 23: Progressive mesh deformation results for the velocity contours 
change in the flow pattern is now possible. This would allow to minimise the number of deformation steps obtaining an eroded state of the geometry in a much shorter period of time. Because of the time it takes to reach convergence after the mesh is deformed, this kind of solver is considered a less efficient approach than calculating averages and deforming the mesh a lower number of times with an increased magnitude of deformation instead.

It is also worth noting that, the smaller the deformation steps and the higher the damage each particle causes on the surface, the more irregular the deformed surface becomes, as evidenced by figure 24. These surfaces were obtained by increasing the damage the particles cause on the surface and deforming the mesh at every time-step, yielding a very uneven geometry at the boundary which eventually caused divergence.

\section{Application to a slurry pump's volute}

Erosion in centrifugal slurry pumps was also investigated and it was found that there is a correlation between the squared of the velocity at the cells nearest to the walls and the erosion ratio for the static parts. A set of applications were implemented in OpenFOAM for calculation of the erosion contours and to deform the mesh according to those. A visual comparison with worn pumps proves the suitability of the technique to calculate performance decay as the pump is being eroded. This technique could also lead to a significant improvement in failure prediction. Figure 25 shows the volute of a centrifugal slurry pump at two different stages. The first two show the pump before mesh deformation with and without erosion contours. The following two show the deformed volute after the algorithm was applied. The volume flow rate of the centrifugal pump in the simulation was $0.235 \frac{\mathrm{m}^{3}}{\mathrm{~s}}$ and its rotational speed was $1100 \mathrm{rpm}$.

Finally, Figure 26 shows the computationally eroded pump next to the volutes of two real slurry pumps. In these images it can be observed how a very good approximation of the erosion pattern found in real volutes can be reproduced with the aid of the proposed methodology. The small differences between 

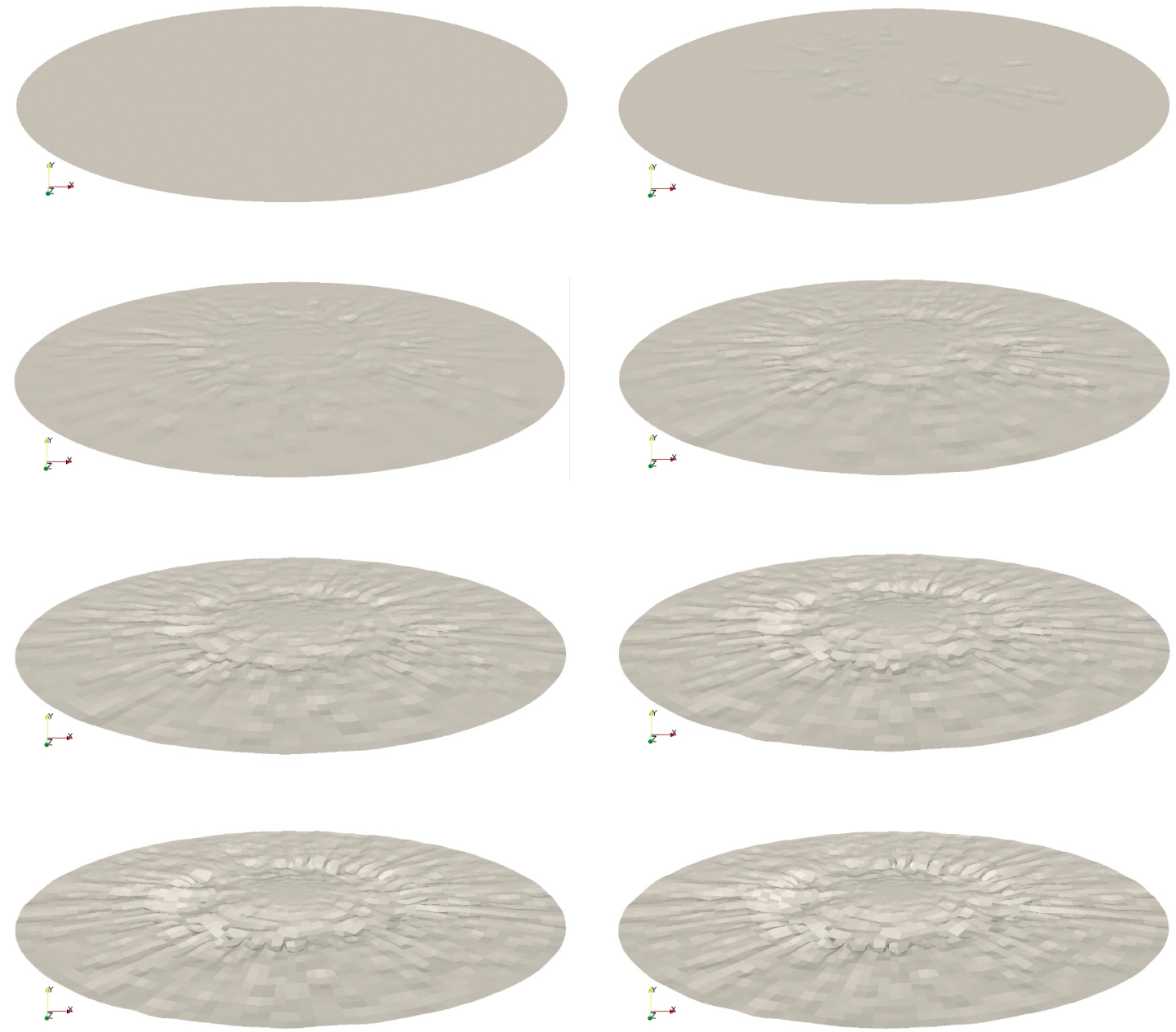

Figure 24: Progressive surface deformation with high damaging particles 

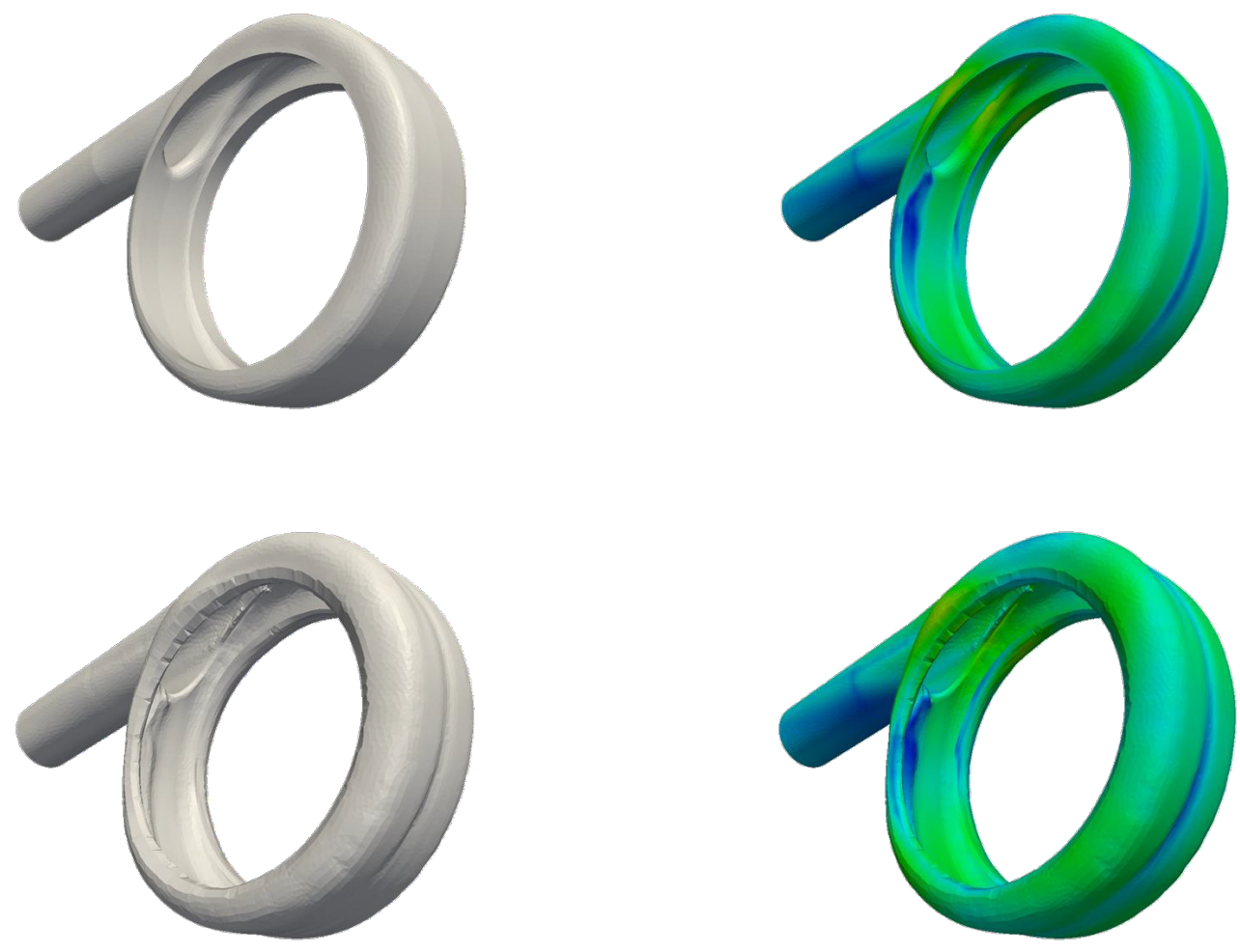

Figure 25: Slurry pump's volute before and after erosion 

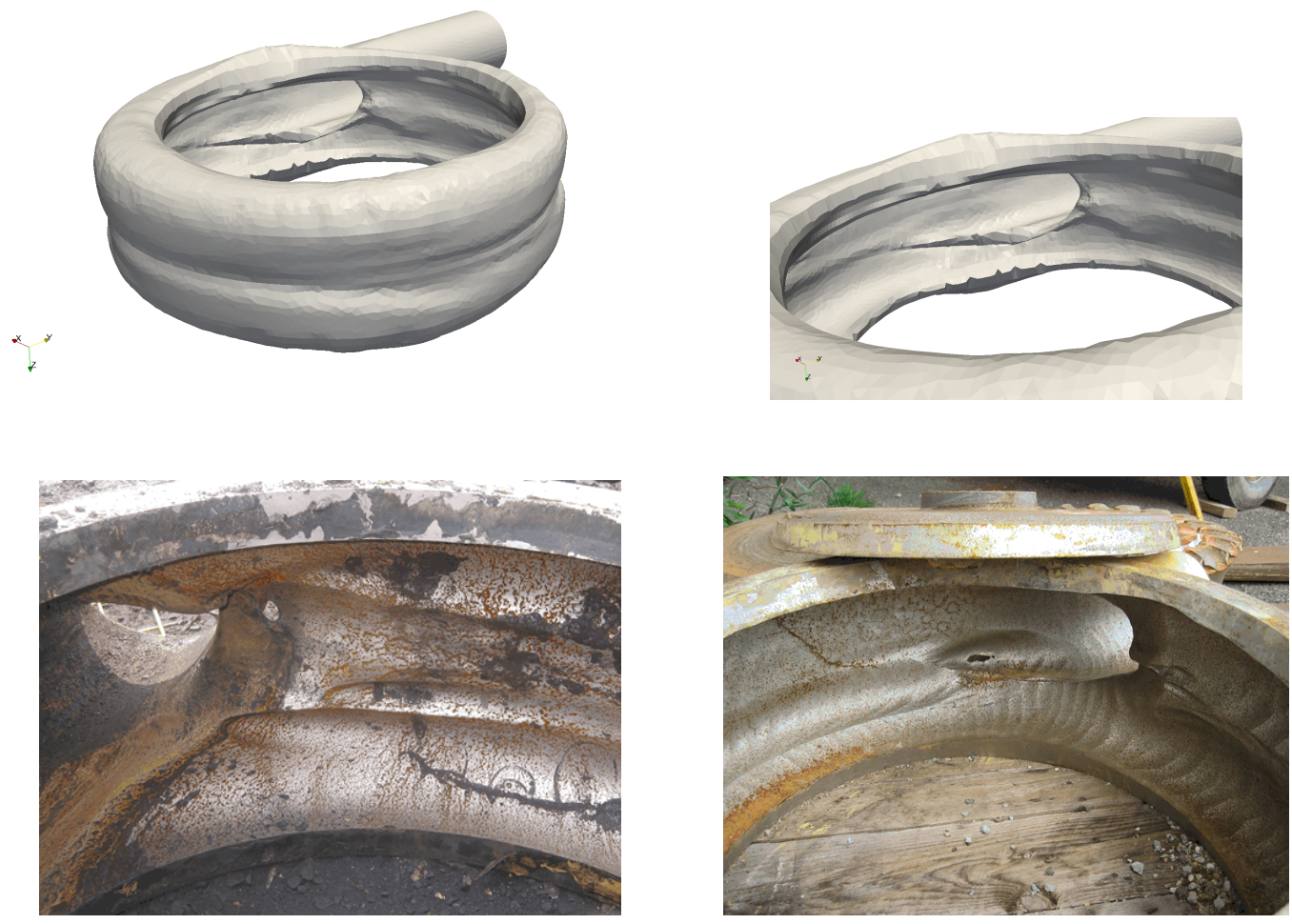

Figure 26: Computationally eroded volute and volutes obtained from pumps operating in the field 
the computational and the field erosion could be due to the position relative to the floor of the pump, as gravity affects the movement of the slurry or small differences in the volume flow rate or rotational speed of the slurry pump.

\section{Conclusions}

A new methodology has been developed for erosion calculation including mesh deformation according to the erosion rate. The mesh deformation algorithm is independent of the geometrical configuration and as such, applicable to any geometry subjected to erosion. With the aid of the implementation of a number of erosion formulae in OpenFOAM, it was proved that, as erosion progresses in the jet impingement, a new stagnation ring appears around the wear scar as the velocity increases induced by the higher bend the fluid experiences. As opposed to Nguyen et al [6], in this work, the deformed surface was obtained only by computational means. The algorithm was also applied to a centrifugal slurry pump's volute and the results compared well to real eroded pumps obtained from some of the clients of The Weir Group PLC. The deformation algorithm can also be used in combination with dynamic meshing for many other applications such as simulating progressive pipe blockage or even the blood flow in arteries progressively blocked by accumulating bodies. In

these cases, the direction of the surface normal vectors would be inverted as the geometry represented by the fluid path would be becoming smaller instead of bigger. Deformation can be coupled not only to erosion but also to other fields such as pressure or velocity to simulate other types of processes.

\section{Acknowledgement}

Results were obtained using the EPSRC funded ARCHIE-WeSt High Performance Computer (www.archie-west.ac.uk). EPSRC grant no. EP/K000586/1. The funding provided by The Weir Group PLC is gratefully acknowledged, along with the valuable contributions of Dr. Luis Moscoso. 


\section{References}

[1] S. H. Kenichi Sugiyama, Kenji Harada, Influence of impact angle of solid particles on erosion by slurry jet, Wear 265 (2008) 713-20.

[2] A. G. et al., An integrated methodology for predicting material wear rates due to erosion, Wear 267 (2009) 1935-44.

[3] A. G. et al., An investigation of a geometry independent integrated method to predict erosion rates in slurry erosion, Wear 271 (2011) 712-9.

[4] A. Lopez, M. Stickland, W. Dempster, W. Nicholls, CFD study of Jet Impingement Test erosion using Ansys Fluent and OpenFOAM, Computer Physics Communications 197 (2015) 88-95.

[5] A. Mackenzie, A. Lopez, K. Ritos, M. Stickland, W. Dempster, A comparison of CFD software packages' ability to model a submerged jet, Eleventh International Conference on CFD in the Minerals and Process Industries, CSIRO, Melbourne, Australia .

[6] V. N. et al, A combined numerical-experimental study on the effect os surface evolution on the water-sand multiphase flow characteristics and the material erosion behavior, Wear 319 (2014) 96-109.

[7] K. C. L. H. C. Meng, Wear models and predictive equations: their form and content, Wear 181 (1995) 443-57.

[8] I. M. Hutchings, A model for the erosion of metals by spherical particles at normal incidence, Wear 70 (1981) 269-81.

[9] R. M. Davies, The determination of static and dynamic yield stresses using a step ball, Proceedings of the Royal Society of London 197A (1949) 416432.

[10] J. G. A. B. A.J. Van Riemsdijk, Erosion in gas-solid systems, Fifth World Petroleum Congress, Section VII, Engineering, Equipment and Materials (1959) 43-58. 
[11] G. G., T. W., An experimental investigation of the erosion characteristics of 2024 aluminum alloy, Department of Aerospace Engineering Tech. Rep., University of Cincinnati (1973) 73-7.

[12] J. G. A. Bitter, A study of erosion phenomena Part I, Wear 6 (1963) 5-21.

[13] J. G. A. Bitter, A study of erosion phenomena Part II, Wear 6 (1963) $169-90$.

[14] J. H. Neilson, A. Gilchrist, Erosion by a stream of solid particles, Wear 11 (1968) 111-22.

[15] L. Ristroph, M. N. J. Moore, S. Childress, M. J. Shelley, J. Zhang, Sculpting of an erodible body by flowing water, PNAS, Proceedings of the National Academy of Sciences 109 (48) (2012) 19606-9.

[16] M. N. J. Moore, L. Ristroph, S. Childress, J. Zhang, M. J. Shelley, Selfsimilar evolution of a body eroding in a fluid flow, Physics of Fluids 25 (2-13) 116602(1-25).

[17] W. H. Mitchell, S. Spagnolie, A generalized traction integral equation for Stokes flow, with applications to near-wall particle mobility and viscous erosion, Journal of Computational Physics 333 (2017) 462-82.

[18] J. N. Hewett, M. Sellier, Evolution of an eroding cylinder in single and lattice arrangements, Journal of Fluids and Structures 70 (2017) 295-313.

[19] S. Rajahram, T. Harvey, R. Wood, Evaluation of a semi-empirical model in predicting erosion-corrosion, Wear 267 (2009) 1883-93.

[20] W. Tabakoff, R. Kotwal, A. Hamed, Erosion study of different materials affected by coal ash particles, Wear 52 (1979) 161-73.

[21] B. E. Launder, D. Spalding, Lectures in Mathematical Models of Turbulence, Academic Press, 1972. 
[22] K. Abdellatif et al, Predicting initial erosion during the hole erosion test by using turbulent flow CFD simulation, Applied Mathematical Modellling 36 (2012) 3359-70.

[23] F. Menter, Two-Equation Eddy-Viscosity Turbulence Models for Engineering Applications, AAIA Journal 32 (1994) 1598-605.

[24] Ansys Inc., Ansys Fluent User Guide, Ansys, Inc., 2009.

[25] P. A. Vesilind, The Rosin-Rammler particle size distribution, Resource recovery and conservation 5 (1980) 275-7.

[26] P. Mathews, Sample Size Calculations: Practical Methods for Engineers and Scientists, Mathews Malnar and bailey, Inc., 2010.

[27] A. Forder, M. Thew, D. Harrison, A numerical investigation of solid particle erosion experienced within oilfield control valve, Wear 216 (1998) 184--193.

[28] E. Huttunen-Saarivirta, H. Kinnunen, J. Turiemo, Erosive wear of boiler steels by sand and ash, Wear 317 (2014) 213-24.

[29] W. Head, M. Harr, The development of a model to predict the erosion of materials by natural contaminants, Wear 15 (1970) 1-46.

[30] M. Menguturk, E. F. Sverdrup, Calculated tolerance of a large electric utility gas turbine to erosion damage by coal gas ash particles, ASTM Special Technical Publications 664 (19) 193-224.

[31] K. Sun, L. Lu, H. Jin, Modeling and numerical analysis of the solid particle erosion in curved ducts, Abstract and Applied Analysis 2013 (2013) 1-8.

[32] X. Song, J. Z. Lin, J. Zhao, T. Y. Shen, Research on reducing erosion by adding ribs on the wall in particulate two-phase flows, Wear 193 (1996) $1-7$.

[33] K. Nandakumar et al, A phenomenological model for erosion of material in a horizontal slurry pipeline flow, Wear 269 (2010) 190-6. 
[34] D. Shepard, A two-dimensional interpolation function for irregularly spaced data, Proceedings 1968 ACM National Conference 1 (1968) 517-24.

[35] G.Allasia, Some physical and mathematical properties of inverse distance weighted methods for scattered data interpolation, Calcolo 29 (1992) 97109. 